\title{
Negative filament tension in the Luo-Rudy model of cardiac tissue
}

\author{
S. Alonso \\ Abteilung Physikalische Chemie, Fritz-Haber-Institut der Max-Planck-Gesellschaft, Faradayweg 4-6, \\ 14195 Berlin, Germany
}

\author{
A. V. Panfilov \\ Theoretical Biology, Utrecht University, Padualaan 83584 CH, Utrecht, The Netherlands
}

(Received 28 July 2006; accepted 8 December 2006; published online 30 March 2007)

\begin{abstract}
Scroll waves are vortices that occur in three-dimensional excitable media. Scroll waves have been observed in a variety of systems including cardiac tissue, where they are associated with cardiac arrhythmias. The disorganization of scroll waves into chaotic behavior is thought to be the mechanism of ventricular fibrillation, which lethality is widely known. One of the possible mechanisms of scroll wave instability is negative filament tension, which was studied theoretically using lowdimensional models of excitable medium. In this article we perform a numerical study of negative filament tension using the Luo-Rudy phase 1 model, which is widely used in cardiac electrophysiology. We show that this instability exists in this model, study its manifestation and discuss its relation to cardiac arrhythmogenesis. (ㅇ 2007 American Institute of Physics.
\end{abstract}

[DOI: $10.1063 / 1.2430638]$

Rotating spiral waves occur in a variety of nonlinear excitable media. The appearance and multiplication of spiral waves disorders the spatial organization of the medium and may result in turbulent or chaotic behavior. If such a regime occurs in cardiac tissue it causes cardiac fibrillation, which is one of the main causes of death in the industrialized world. ${ }^{1}$ Several mechanisms were proposed to explain the origin of the instability which produces this turbulence. The most discussed of them relate the instability to tissue heterogeneities or to dynamical instabilities of wave propagation in cardiac tissue. In this article we investigate an alternative mechanism based on negative filament tension of scroll waves in cardiac tissue. We show that negative filament tension occurs in a one of the most widely used ionic models of cardiac tissue, the Luo-Rudy phase 1 model, determine the region of parameters for this instability, and discuss its possible application for cardiac arrhythmias in conditions of low excitability.

\section{INTRODUCTION}

Contraction of the heart is controlled by electrical waves of excitation that propagate through the myocardium and induce the contraction of the cardiac fibers. Abnormal excitation of the heart results in cardiac arrhythmias. Ventricular tachycardia is a common cardiac arrhythmia that is characterized by a high rate of cardiac excitation and thus high rate of contraction of the heart. Experimental and clinical studies show that in most of the cases tachycardia is driven by a reentrant wave of excitation, also called spiral wave. Spiral waves have been found in a wide variety of nonlinear systems in physics, chemistry, and biology. ${ }^{2}$ Ventricular tachycardia can deteriorate into ventricular fibrillation (VF). VF is characterized by a turbulent pattern of excitation that disrupts the coordinated contraction of the heart and results in sudden cardiac death. Currently, the two main hypothesis of VF are the mother rotor and the multiple wavelet hypotheses. The mother rotor hypothesis assumes that VF is driven by one stable high-frequency source (mother rotor), and complex VF patterns are the result of multiple breaks of the waves from this source at heterogeneities of the heart. ${ }^{3}$ The multiple wavelet hypothesis explains VF by breakup of spiral waves due to dynamical or anatomical heterogeneity., Many possibilities for spiral breakup have been studied theoretically. ${ }^{6}$ However, the exact mechanism of onset of this chaotic pattern in the heart remains unknown.

There are several factors that are considered important for $\mathrm{VF}$ onset. One of such factors is the three-dimensional (3D) structure of the heart. As was discussed in Ref. 7, VF can occur if the thickness of the ventricular wall of the heart exceeds some critical value; thus, it implies that instabilities of waves in three dimensions may be of great importance.

The extension of a spiral wave into three dimensions is a scroll wave. Scroll waves have been observed in many excitable systems, such as the Belousov-Zhabotinsky chemical reaction, ${ }^{8,9}$ in the slug phase of the life cycle of slime molds, ${ }^{10}$ and in the ventricles of the heart during cardiac arrhythmias. ${ }^{11,12}$ Numerous modeling studies of scroll waves have been performed using analytical and numerical methods. The importance of scroll waves for our understanding of the behavior of excitable media has been consistently emphasized in the literature. ${ }^{13}$

A scroll wave is usually characterized by its filament, ${ }^{2}$ which is an extension into three dimensions of the notion of the core of spiral waves. The filament can be regarded as a union of the cores of all the spirals, which can be seen in various sections of the scroll wave. This filament can be straight or curved, or can even be closed into a ring. This last type of waves with a circular filament are known as scroll rings (Fig. 1). Scroll ring filaments are nonstationary and 


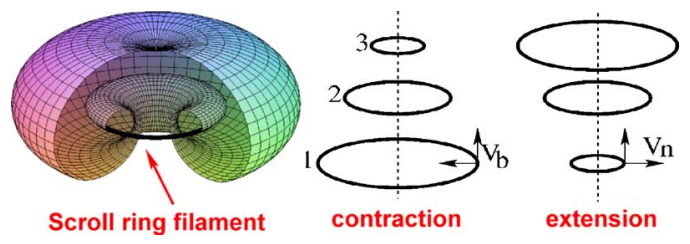

FIG. 1. Schematic representation of a scroll ring and its filament for the two main dynamics of filament drift: contraction and extension regimes.

drift in space (Fig. 1). The two main drift regimes are filament contraction (positive tension) and filament expansion (negative tension). ${ }^{14}$ Drift can be characterized by the velocity, which has components in the normal and binormal directions. Both drift velocities in the normal $V_{n}$ (horizontal in Fig. 1) and $V_{b}$ the binormal (vertical) are proportional to the curvature $k$ of the filament:

$$
V_{n}=D_{n} k
$$

$$
V_{b}=D_{b} k,
$$

where $D_{n}$ and $D_{b}$ are the proportionality coefficients. These relations have been verified in $3 \mathrm{D}$ experiments with the Belousov-Zhabotinsky reaction, where scroll rings are observed to shrink with a rate proportional to the curvature.

Filament tension is important for filament stability. ${ }^{15}$ If the tension of the filament is positive, the filament tends to become shorter, which results either in the collapse of the scroll wave with a closed (circular) filament or in the stabilization of a straight filament between two boundaries. ${ }^{16}$ If the filament tension is negative this will result in an instability because the filament will tend to increase its length and as a result there will be the formation of new filaments when the growing filament touches the boundaries of the medium. The negative filament tension has been associated to a translation band instability. ${ }^{17}$ Although negative filament tension still has not been observed in experiments, it was studied analytically in Ref. 18 and observed in numerical simulations in the FitzHugh-Nagumo model Refs. 14 and 15 and in the Fenton-Karma model. ${ }^{6}$ Later, numerical studies on the final complex regime, were performed ${ }^{19,20}$ with the Barkley model of excitable media. ${ }^{21}$

In spite of potential importance of negative filament tension for VF, this type of instabilities has not been studied in any ionic model for cardiac tissue that incorporates the most accurate description of properties of cardiac cells. Currently there are many ionic models for cardiac cells which describe various types of cardiac tissue (for a review, see Ref. 23). However, for 3D cardiac simulations the most widely used is the Luo-Rudy model for ventricular cardiac cells. ${ }^{22,24}$ In this paper we use the Luo-Rudy model to study negative filament tension. We show that negative filament tension can occur in this model, find the parameter region for its onset and give a two-dimensional (2D) test that may help to identify this phenomenon in cardiac tissue. We also discuss its possible applicability to experimental research.

\section{METHODS}

Cardiac tissue can be modeled by the following system of parabolic partial differential equations: ${ }^{25,26}$

$$
\frac{\partial V}{\partial t}=-\frac{I_{\text {ion }}}{C_{m}}+D \nabla^{2} V .
$$

Here, $V$ is the transmembrane potential, $t$ is time, $C_{m}$ $=1 \mu \mathrm{F} / \mathrm{cm}^{2}$ is the membrane capacitance, $D$ $=0.001 \mathrm{~cm}^{2} / \mathrm{ms}$ is the diffusion coefficient, and $I_{\text {ion }}$ is the sum of all transmembrane ionic currents. We use the Luo-Rudy phase 1 model (LR1) ${ }^{22}$ for the calculation of the total current: $I_{\text {ion }}=I_{\mathrm{Na}}+I_{\mathrm{Si}}+I_{K}+I_{K 1}+I_{K p}+I_{b}$, where $I_{\mathrm{Na}}$ $=G_{\mathrm{Na}} m^{3} h j\left(V-E_{\mathrm{Na}}\right)$ is the fast $\mathrm{Na}^{+}$current, $I_{\mathrm{Si}}=G_{\mathrm{Si}} d f(V$ $\left.-E_{\mathrm{Si}}\right)$ is the slow inward $\mathrm{Ca}^{2+}$ current, $I_{K}=G_{K} x x_{1}\left(V-E_{K}\right)$ is the slow outward $\mathrm{K}^{+}$current, $I_{K 1}=G_{K 1} K 1_{\infty}\left(V-E_{K 1}\right)$ is the time-independent $\mathrm{K}^{+}$current, $I_{K p}=G_{K p} K_{p}\left(V-E_{K p}\right)$ is the plateau $\mathrm{K}^{+}$current, and $I_{b}=G_{b}\left(V-E_{b}\right)$ is the background current. The variables $m, h, j, d, f$, and $x$ are gating variables, the dynamics of which can be modeled by

$$
\frac{d y}{d t}=\left(y_{\infty-y}\right) / \tau_{y},
$$

where $y$ represents any of the gating variables. We use the following ionic concentrations: $\left[\mathrm{Na}^{+}\right]_{i}=18 \mathrm{mM},\left[\mathrm{Na}^{+}\right]_{o}$ $=140 \mathrm{mM},\left[\mathrm{K}^{+}\right]_{i}=145 \mathrm{mM}$ and $\left[\mathrm{K}^{+}\right]_{o}=5.4 \mathrm{mM}$, the maximal conductances for the potassium currents are $G_{K}$ $=0.282 \mathrm{mS} / \mathrm{cm}^{2}$ and $G_{K 1}=0.6047 \mathrm{mS} / \mathrm{cm}^{2}$, and the maximal conductances for sodium and inward slow calcium currents are, respectively, $G_{\mathrm{Na}}=23.0 \mathrm{mS} / \mathrm{cm}^{2}$ and $G_{\mathrm{Si}}=0.09 \mathrm{mS} / \mathrm{cm}^{2}$. The values of $G_{\mathrm{Na}}, G_{\mathrm{Si}}$ and $\left[\mathrm{K}^{+}\right]_{o}$ are varied and will be specified later. For more details of the model, see Ref. 22.

We numerically integrate Eq. (3) by the Euler method with a temporal step $\Delta t=0.02 \mathrm{~ms}$ and by the use of finite differences for the calculation of the Laplacian with a spatial step $\Delta x=0.02 \mathrm{~cm}$. We solve Eq. (3) in a one-dimensional cable in order to calculate the velocity and the duration of the action potential (APD). The velocity of the traveling wave is calculated once the shape of the action potential is stationary. The APD is defined by the condition $V>-72 \mathrm{mV}$ when the resting potential is around $V_{R}=-84 \mathrm{mV} .^{27,28}$ However, the resting potential depends on the concentration $\left[\mathrm{K}^{+}\right]_{o}$; therefore, for larger values of the concentration of extracellular potassium, we approximate the APD by the condition $V>\left(V_{R}+10\right) \mathrm{mV}$. Spirals waves are generated in $2 \mathrm{D}$ domains of up to $14 \times 14 \mathrm{~cm}^{2}$ by applying the $\mathrm{S} 1-\mathrm{S} 2$ protocol. $^{25,26}$ Scroll waves are generated from a stack of 2D spiral waves by displacement of the solutions along the vertical axis with a sinusoidal perturbation of the pattern in order to create an initial perturbation on the scroll filament. The size of the tissue is up to $4 \times 4 \times 4 \mathrm{~cm}^{3}$. We used such large tissue size in order to minimize possible effects of the boundaries on our solutions. We used no-flux boundaries conditions.

The radial symmetry of scroll rings allows us to study filament drift using just two spatial coordinates. ${ }^{14}$ Indeed, for the Laplacian of Eq. (3) in a cylindrical coordinate system $r, \phi, z$, all angular derivatives will disappear, due to the symmetry of the problem, and the equation can be rewritten as 

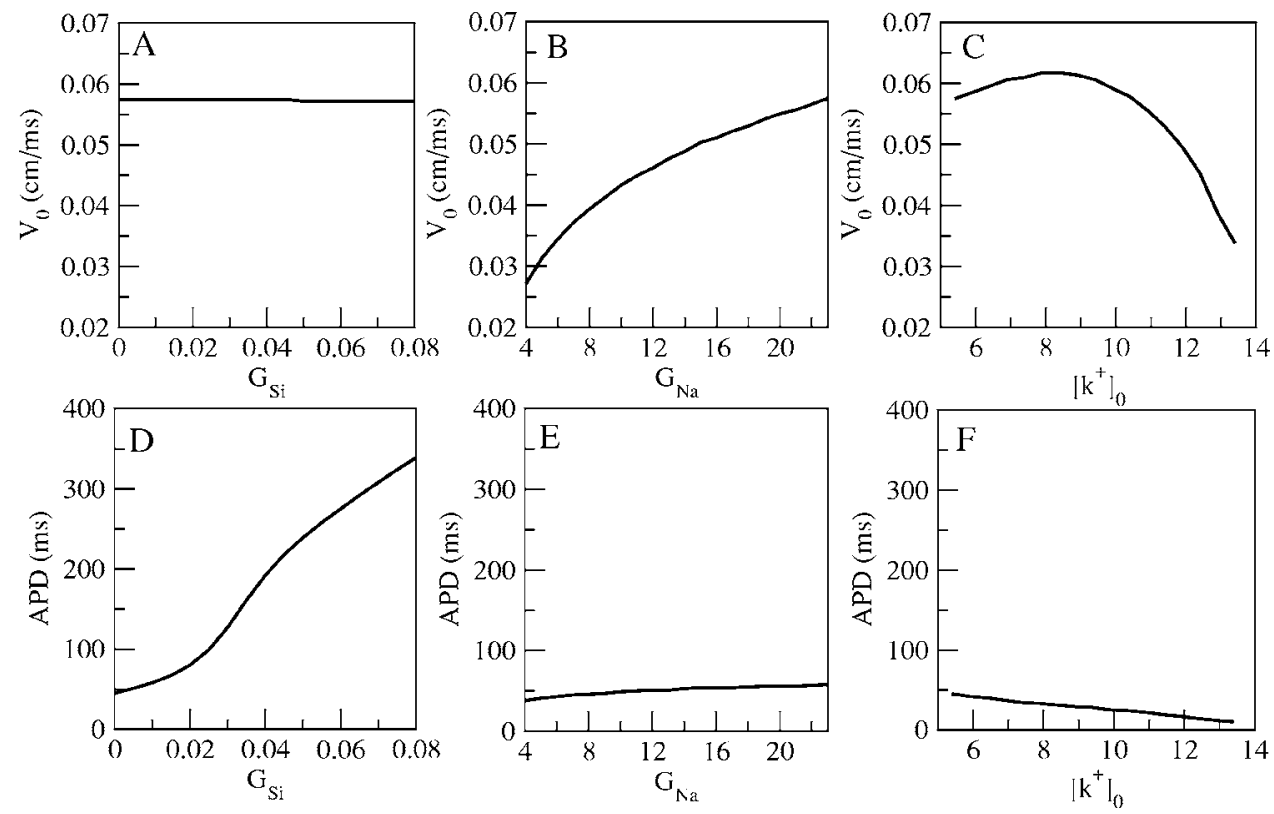

FIG. 2. Velocity and APD of a traveling wave propagating in cardiac tissue for different values of $G_{\mathrm{Na}}, G_{\mathrm{Si}}$, and $\left[\mathrm{K}^{+}\right]_{o}$. The values $\left[\mathrm{K}^{+}\right]_{o}=5.4 \mathrm{mM}$, and $G_{\mathrm{Na}}=23.0 \mathrm{mS} / \mathrm{cm}^{2}$ are kept constant for panels (A) and (D), $\left[\mathrm{K}^{+}\right]_{0}$ $=5.4 \mathrm{mM}$ and $G_{\mathrm{Si}}=0.01 \mathrm{mS} / \mathrm{cm}^{2}$ for panels (B) and (E), and $G_{\mathrm{S}}$ $=0.0 \mathrm{mS} / \mathrm{cm}^{2}$ and $G_{\mathrm{Na}}=23.0 \mathrm{mS} / \mathrm{cm}^{2}$ for panels $(\mathrm{C})$ and $(\mathrm{F})$.

$$
\frac{\partial V}{\partial t}=-\frac{I_{\text {ion }}}{C_{m}}+D\left(\frac{\partial^{2}}{\partial r^{2}}+\frac{1}{r} \frac{\partial}{\partial r}+\frac{\partial^{2}}{\partial z^{2}}\right) V .
$$

The trajectories of the spiral tip and the dynamics of the filaments of the scroll wave are found using the algorithm of filament tracing developed in Ref. 29 at the level of $V=-35 \mathrm{mV}$.

\section{RESULTS}

\section{A. Weakly excitable cardiac tissue in the LR1 model}

In low-dimensional models of excitable media negative filament tension was found under conditions of weak excitability where the motion of the waves is quite stable and the front-tail interaction between the consecutive pulses is absent. The main parameters of the LR1 model that affect excitability are the maximum sodium conductance $G_{\mathrm{Na}}$, the maximum conductance of the slow inward current $G_{\mathrm{Si}}$ and the concentration of extracellular potassium $\left[\mathrm{K}^{+}\right]_{o}$. Note that these parameters are also important in the study of acute myocardial ischemia, ${ }^{30}$ which is one of the main physiological conditions that reduces the excitability of cardiac tissue.

We reduce the value of the maximum conductance $G_{\mathrm{Si}}$, which controls the slow inward current. The effect of this modification is shortening of APD. If APD is short there is less interaction between the pulses and, thus, the trajectories of the tip in 2D and of the filament in 3D are more regular. Therefore, we employ low values of $G_{\mathrm{Si}}$, in order to avoid complex meanders and breakup. ${ }^{28}$ Note that the reduction of $G_{\mathrm{Si}}$ was used in Ref. 30 to simulate conditions of acidosis due to ischemia.

Figure 2 shows the effect of these parameters on the main characteristics of the cardiac action potential. We see that the velocity of a traveling wave is almost independent of the value of the slow inward current [Fig. 2(A)]. However, the APD decreases substantially with a decrease of $G_{\mathrm{Si}}$ [Fig. $2(\mathrm{D})]$. The value of $G_{\mathrm{Na}}$ has a substantial effect on the velocity of the waves [Fig. 2(B)], but the APD remains almost

constant [Fig. 2(E)]. Effects of $\left[\mathrm{K}^{+}\right]_{o}$ are more complex: we see a nonmonotonic dependence of the velocity on the potassium concentration [Fig. 2(C)] and slight decrease in APD for large values of $\left[\mathrm{K}^{+}\right]_{o}$ [Fig. $\left.2(\mathrm{~F})\right]$.

With these considerations we reduce excitability in two steps: first, by the reduction of the slow inward current conductance $G_{\mathrm{Si}}$, and second, by the reduction of the maximum sodium current conductance $G_{\mathrm{Na}}$ or the increase of extracellular potassium concentration $\left[\mathrm{K}^{+}\right]_{o}$.

In some simulations, we modify the dynamics of the variable $j$ by clamping it to 1 . The reason for that is the same as for the modification of $G_{\mathrm{Si}}$. As shown in numerical simulations, ${ }^{28}$ the rotation of spiral waves in the LR1 model with clamped values of $j$ is much more stable, and in some cases even rigid rotation of spiral waves is observed. This particular modification of the original model was already considered in the original article of Luo and Rudy, ${ }^{22}$ and it was one of the central assumptions of the Ebihara and Johnson model of the sodium current. ${ }^{31}$

\section{B. Negative filament tension}

We found that negative filament tension can occur in the LR1 model of cardiac tissue in conditions of low excitability. Figure 3 shows typical dynamics.

The simulation starts with an initial condition in the form of a scroll wave with a sinusoidally perturbed straight filament [Fig. 3(A)]. After several rotations the filament begins to bend [Fig. 3(B)] and the length and curvature of the filament increases [Figs. 3(C) and 3(D)] until the filament touches the boundary [Fig. 3(E)] and a new scroll wave appears. This process goes ahead giving rise to a complex spatio-temporal pattern of excitation [Figs. 3(E) and 3(F)]. This chaotic dynamics persists as long as we perform the simulation $(10 \mathrm{~s})$. As shown in Fig. 3, new filaments appear and disappear as a result of the interaction with the boundaries or due to the interaction between the filaments. The 

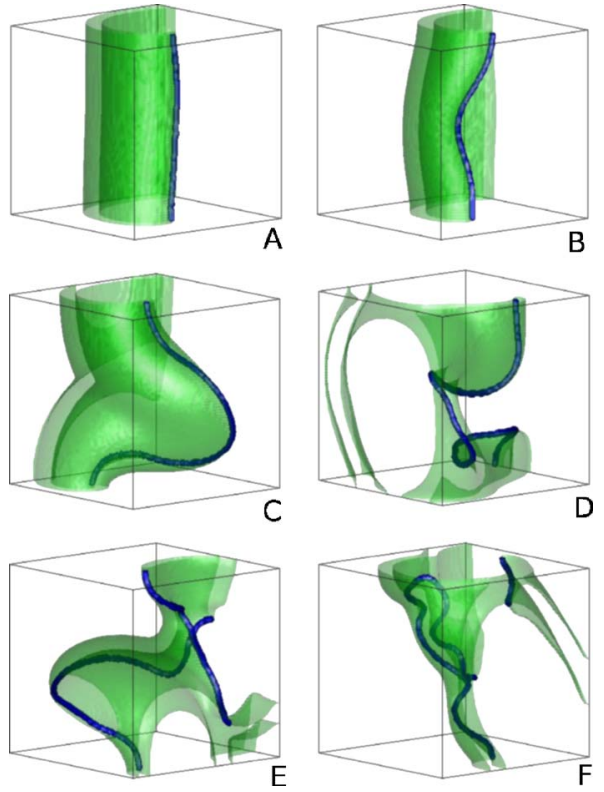

FIG. 3. Evolution of the filament (solid lines) and the waves (gray surfaces) under condition of negative filament tension in the Luo-Rudy model. The parameter values are $G_{\mathrm{Na}}=3.9 \mathrm{mS} / \mathrm{cm}^{2}$ and $G_{\mathrm{Si}}=0.0 \mathrm{mS} / \mathrm{cm}^{2}$. The snapshots correspond to times: (A) $t=200 \mathrm{~ms}$, (B) $t=480 \mathrm{~ms}$, (C) $t=784 \mathrm{~ms}$, (D) $t=1084 \mathrm{~ms}$, (E) $t=1548 \mathrm{~ms}$, and (F) $t=1848 \mathrm{~ms}$.

excitation pattern is similar to the dynamics obtained in low dimensional models of excitable media under negative filament tension. ${ }^{6,15,19}$

The filament instability is a pure three-dimensional effect; spiral waves are completely stable in two dimensions under the same parameter values. It means that there is a minimal thickness of the wall in order to observe the instability. In Fig. 4 we show the results of numerical simulations with the same parameter values as in Fig. 3 but for different vertical thicknesses. For thick three-dimensional tissue the negative tension instability develops [Fig. 4(A)] similar to that in Fig. 3. However, when the tissue is very thin, the instability does not develop and the scroll wave rotates similar to a spiral wave in two-dimensional media [Fig. 4(C)]. For the intermediate thickness the filament is unstable, but it undergoes a complex meandering motion without breaking up [Fig. 4(B)].

In the above-described simulations, the values of the parameters were $G_{\mathrm{Na}}=3.9 \mathrm{mS} / \mathrm{cm}^{2}$ and $G_{\mathrm{Si}}=0.0 \mathrm{mS} / \mathrm{cm}^{2}$. Increasing either $G_{\mathrm{Na}}$ or $G_{\mathrm{Si}}$ results in an increase of the excitability of the cardiac tissue causing the tension of the filament to become positive. For positive filament tension we observed that the initially perturbed filament stabilizes between the opposite boundaries and remains straight for the whole simulation.

In order to find the region in parameter space where scroll rings expand, we performed numerical simulation for different values of $G_{\mathrm{Na}}$, using the radial symmetry assumption [see Eq. (5)]. The results of two simulations of this type are plotted in Fig. 5, where we show the position of the free edge of the scroll ring (analogous to the spiral tip in two dimensions) in the course of time for two different values of the sodium conductance. We see fast oscillations in the $R$ and

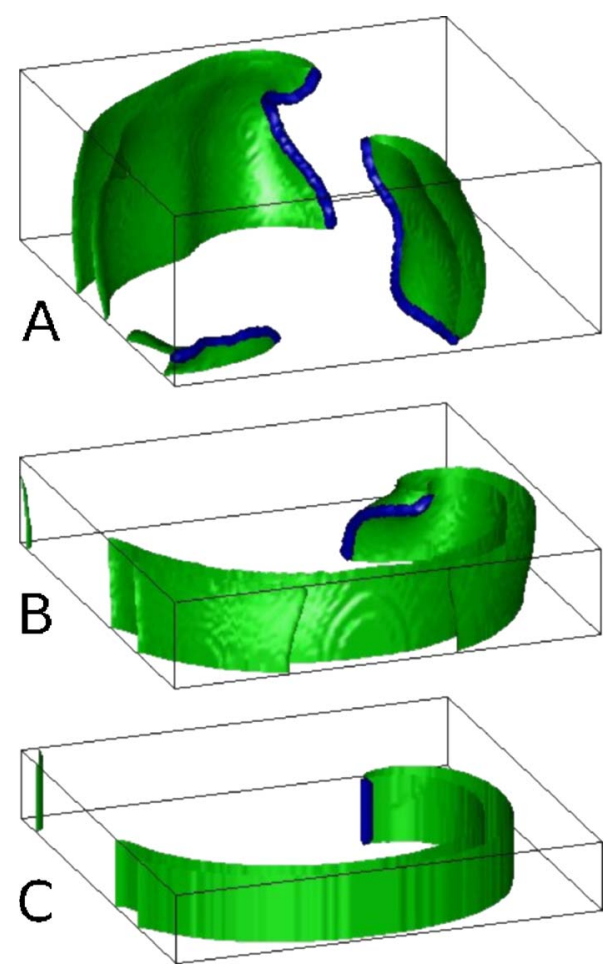

FIG. 4. Evolution of the filament (solid lines) and the waves (gray surfaces) under condition of negative filament tension in the Luo-Rudy model for a slab of tissue of $5 \mathrm{~cm} \times 5 \mathrm{~cm}$ and different thicknesses: $2 \mathrm{~cm}(\mathrm{~A}), 1 \mathrm{~cm}(\mathrm{~B})$, and $0.8 \mathrm{~cm}(\mathrm{C})$. The parameter values are $G_{\mathrm{Na}}=3.9 \mathrm{mS} / \mathrm{cm}^{2}$ and $G_{\mathrm{Si}}$ $=0.0 \mathrm{mS} / \mathrm{cm}^{2}$. The snapshots correspond to times around $t=2 \mathrm{~s}$.

$Z$ values, which reflect the rotation of a scroll ring around a core region that has size of about $1 \mathrm{~cm}$, and slow drift of the filament in space. The left column of Fig. 5, which corresponds to the weak excitability case from Fig. 3, shows the scroll ring with negative filament tension: the scroll ring radius increases with time, which, as we saw in Fig. 3, results in filament instability. The right column shows the results for a larger value of $G_{\mathrm{Na}}$. We see that now the scroll ring has positive filament tension: the radius of the scroll ring decreases, which in 3D corresponds to the case in which straight filaments are stable.

The average drift velocities of the filament in the normal and binormal directions for different $G_{\mathrm{Na}}$ are shown in Fig. 6 . We see [Fig. 6(A)] that for $G_{\mathrm{Na}}<4.10 \mathrm{mS} / \mathrm{cm}^{2}$, the normal drift velocity is positive, corresponding to negative tension filament tension, while for $G_{\mathrm{Na}}>4.16 \mathrm{mS} / \mathrm{cm}^{2}$, the normal drift velocity is negative corresponding to positive filament tension. Interestingly enough, the transition from the positive to the negative filament tension regime is not continuous for both normal and binormal components.

Change of dynamics of spiral wave rotation under the change of $G_{\mathrm{Na}}$ is shown in Fig. 7. In order to avoid Doppler effects, we calculate the average of the periods of the waves calculated near the four corners of the medium. We see that the period decreases with the increase of $G_{\mathrm{Na}}$, but there is a nonmonotonic increase in period around the transition point between positive and negative tension. This period increase is due to meandering of the spirals. Indeed, as shown in Ref. 32 , meandering (under periodic forcing) affects the period of 

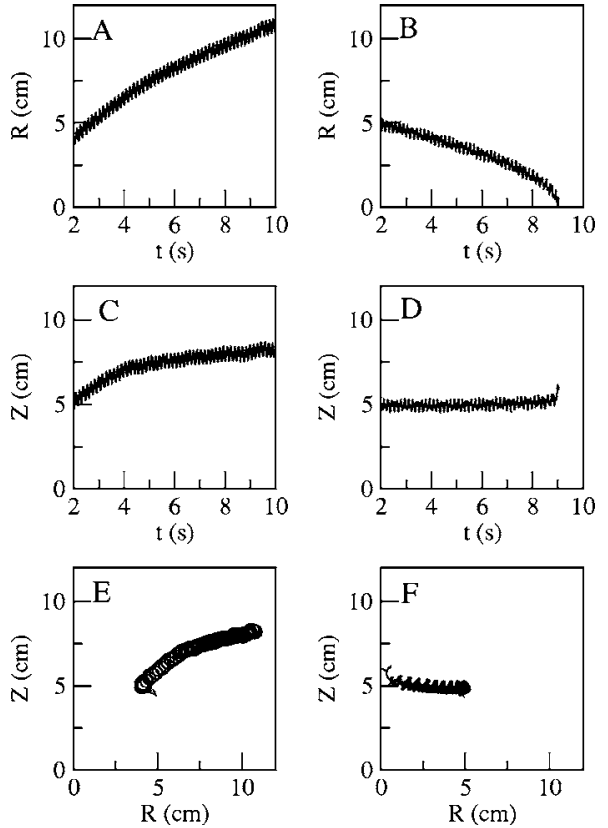

FIG. 5. (Left) Expansion of a scroll ring corresponding to negative filament tension $\left(G_{\mathrm{Na}}=3.9 \mathrm{mS} / \mathrm{cm}^{2}\right.$ and $\left.G_{\mathrm{Si}}=0.0 \mathrm{mS} / \mathrm{cm}^{2}\right)$. (Right) Collapse of a scroll ring corresponding to positive filament tension $\left(G_{\mathrm{Na}}=5.0 \mathrm{mS} / \mathrm{cm}^{2}\right.$ and $\left.G_{\mathrm{Si}}=0.0 \mathrm{mS} / \mathrm{cm}^{2}\right)$. The evolution of the radius $(\mathrm{A}, \mathrm{B})$ and of the vertical coordinate $(\mathrm{C}, \mathrm{D})$, and the dynamics in the plane $(R, Z)(\mathrm{E}, \mathrm{F})$ are plotted.

spiral wave rotation. The variance of the period, which is closely related to the amplitude of the meandering, also increases around the transition point. We see substantial changes in the meandering pattern of spiral waves with the increase of $G_{\mathrm{Na}}$. As the core shape changes from a circular core to inward loop meandering, the radius of the meandering pattern increases dramatically (around $G_{\mathrm{Na}}=4 \mathrm{mS} / \mathrm{cm}^{2}$ ). Further increase in $G_{\mathrm{Na}}$ results in change of the meandering regime from inward to outward loops. For larger values of $G_{\mathrm{Na}}$ there is a decrease of the meandering radius of this outward loop pattern. We also see that the transition boundary
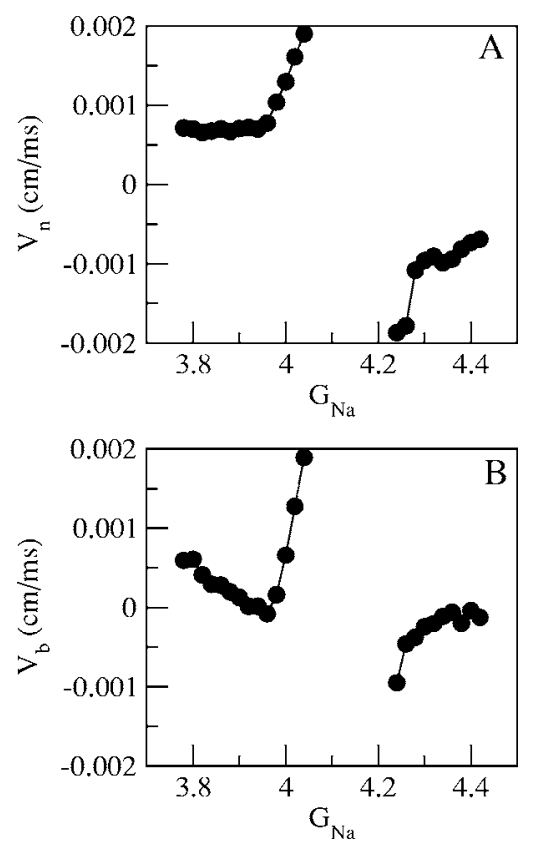

FIG. 6. Value of the normal (A) and binormal (B) velocities of the filament of scroll rings for different values of the excitability. The parameter $G_{\mathrm{Si}}$ $=0.01 \mathrm{mS} / \mathrm{cm}^{2}$ is kept constant.

between positive and negative filament tension coincides with the boundary between inward and outward loops of the meandering motion.

Now we can explain the noncontinuity of the velocities in Fig. 6. Such noncontinuity occurs in the region where meandering of spirals has a large radius. In this region, the drift pattern is determined mainly by the meandering and not by $3 \mathrm{D}$ effects. In addition, in that region, spiral waves typically disappear as a result of the collision with the boundaries.

A two parametric study of filament drift is shown in Fig. 8 . Here, the evolution of the filaments in the plane $(R, Z)$ are plotted for different values of $G_{\mathrm{Na}}$ and $G_{\mathrm{Si}}$. All the scroll

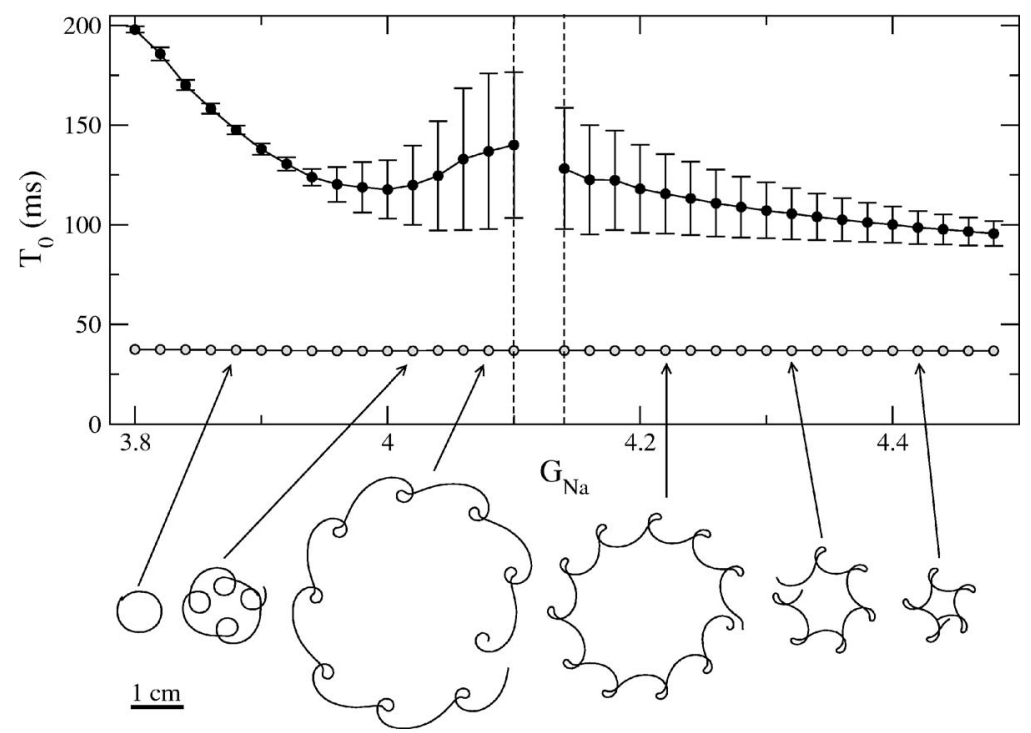

FIG. 7. Period of spiral wave (black points) and the corresponding meandering patterns for different values of the excitability, the value of the parameters are the same as in the previous figure. Error bars show the dispersion of the period, the grey points below the period graph show APD, which does not change substantially for these parameter values. At the bottom, the meandering patterns corresponding to $G_{\mathrm{Na}}$ $=3.88 \mathrm{mS} / \mathrm{cm}^{2}, \quad G_{\mathrm{Na}}=4.02 \mathrm{mS} / \mathrm{cm}^{2}, \quad G_{\mathrm{Na}}=4.08$ $\mathrm{mS} / \mathrm{cm}^{2}, G_{\mathrm{Na}}=4.22 \mathrm{mS} / \mathrm{cm}^{2}, G_{\mathrm{Na}}=4.32 \mathrm{mS} / \mathrm{cm}^{2}$ and $G_{\mathrm{Na}}=4.42 \mathrm{mS} / \mathrm{cm}^{2}$. 


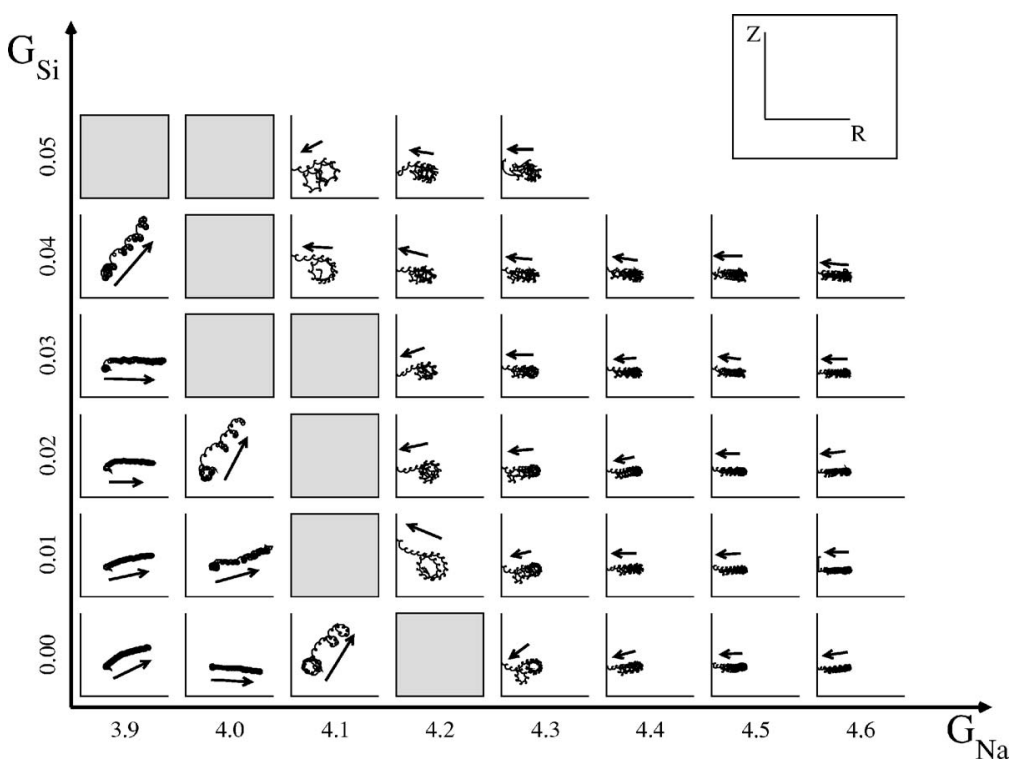

FIG. 8. Phase diagram of the motion of the filament of scroll rings in the plane $(R, Z)$ in front of the variables $G_{\mathrm{Na}}$ and $G_{\mathrm{Si}}$. The evolution of the filament is plotted in all the panels for $10 \mathrm{~s}$. The arrows show the direction of the motion.

rings begin with the same initial radius and the direction of motion is shown by an arrow. Note, that decreasing of $G_{\mathrm{Na}}$ $<3.6 \mathrm{mS} / \mathrm{cm}^{2}$ results in propagation failure and cardiac tissue becomes nonexcitable. We see that for small values of $G_{\mathrm{Na}}$ scroll rings expand and this dynamics corresponds to negative filament tension. For large values of $G_{\mathrm{Na}}$ scroll rings collapse and disappear, corresponding to positive filament tension. In the region between extension and contraction of scroll rings (gray squares in Fig. 8), the drift pattern was more complex due to pronounced meandering of spiral waves in a way similar to that discussed above.

In order to relate filament drift to the corresponding dynamics of spiral waves we performed 2D simulations for the same value of the parameters. The results are shown in Fig. 9. We see that for low values of $G_{\mathrm{Na}}$ and $G_{\mathrm{Si}}$ spiral waves rotate rigidly around the central core. When the value of $G_{\mathrm{Na}}$ is increased the spiral wave develops a complex motion composed by two frequencies and meandering of spiral waves with inward loops is observed. For larger values of $G_{\mathrm{Na}}$ the spiral waves also meander but now with outward loops. Be- tween both regimes straight drift of spiral waves can be observed. ${ }^{33}$ We also see that for increasing values of $G_{\mathrm{Si}}$, meandering is different because the spiral performs large straight motions between the loops of the meandering. For large values of $G_{\mathrm{Si}}$, spiral waves break up, and the study of tip dynamics is no longer possible.

The comparison of Figs. 8 and 9 shows a clear relation between the meandering with inward loops in 2D and the negative filament tension in $3 \mathrm{D}$; also between meandering with outward loops in 2D and the positive filament tension in 3D. This relation has also been observed in low dimensional models of excitable media and cardiac tissue, ${ }^{6,34}$ and in the Oregonator model of the Belousov-Zhabotinsky reaction. ${ }^{35}$ Here we show that it also works for a detailed model of cardiac tissue. It allows us to propose a $2 \mathrm{D}$ test for negative filament tension: we need to study meandering of spiral waves in 2D slices of cardiac tissue. Negative filament tension can be expected for such conditions in cardiac tissue that produce an inward loop meandering of spiral waves.

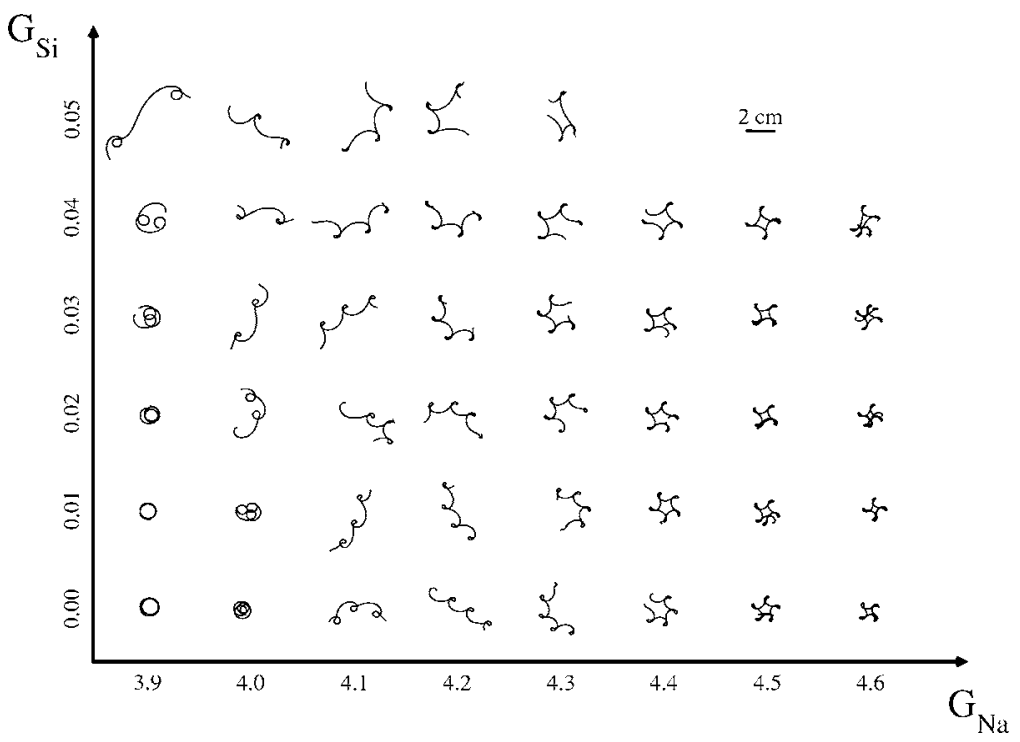

FIG. 9. Phase diagram of the motion of the tip of spiral waves in $2 \mathrm{D}$ cardiac tissue in front of the variables $G_{\mathrm{Na}}$ and $G_{\mathrm{Si}}$. The evolution of the tip is plotted in all the panels for $200 \mathrm{~ms}$. 


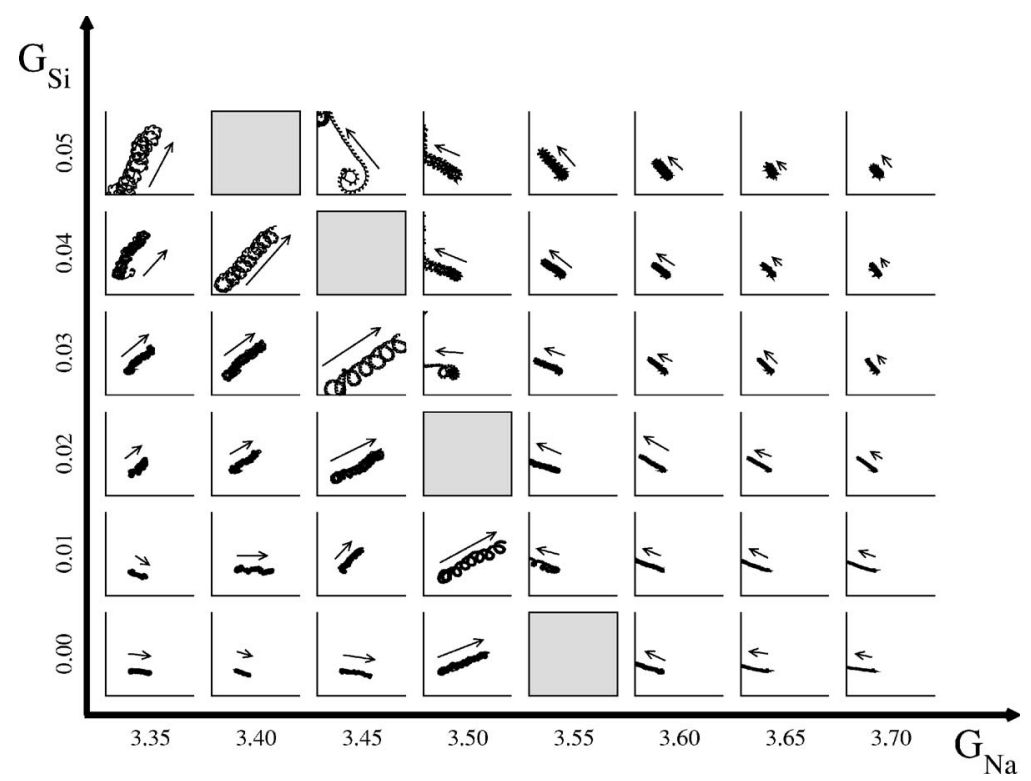

FIG. 10. Phase diagram of the motion of the filament of scroll rings in the plane $(R, Z)$, with $j$ clamped to 1 , in front of the variables $G_{\mathrm{Na}}$ and $G_{\mathrm{Si}}$, keeping $G_{K}$ $=0.423 \mathrm{mS} / \mathrm{cm}^{2}$. The evolution of the filament is plotted in all the panels for $10 \mathrm{~s}$. The arrows show the direction of the motion.

We have also studied how the modification of sodium current by clamping of the gate variable $j$ to 1 affects filament drift. Clamping of the $j$ gate to 1 substantially reduces the meandering of spiral waves. ${ }^{28}$

Similar to the previous figures, Figs. 10 and 11 show the filament drift and the corresponding meandering patterns for different values of $G_{\mathrm{Na}}$ and $G_{\mathrm{Si}}$. We see that meandering of spiral waves with $j$ clamped to 1 is less pronounced and is similar to the meandering observed in low-dimensional models of excitable media near the condition of weak excitability.

The main 3D effect of the clamping of $j$ is a shift of the boundary between positive and negative filament tension toward larger values of $G_{\mathrm{Na}}$ and a change of the direction of the vertical drift of the scroll rings in Figs. 8 and 10: the binormal velocity of the contracting scroll rings in Fig. 8 is positive for large values of $G_{\mathrm{Si}}$ and negative for small values of $G_{\mathrm{Si}}$, while in Fig. 10 the contracting scroll rings always drift upward.

\section{Increased extracellular potassium concentration $\left[\mathrm{K}^{+}\right]_{0}$}

One of the main physiological conditions resulting in a decrease of excitability of cardiac tissue is ischemia. In the LR1 model the effects of ischemia are usually represented by the decrease of the sodium and calcium conductances $\left(G_{\mathrm{Na}}\right.$ and $\left.G_{\mathrm{Si}}\right),{ }^{30}$ by the decrease of cell-cell coupling in the diffusion coefficient term, ${ }^{37}$ and mainly by elevation of the level of extracellular potassium $\left[\mathrm{K}^{+}\right]_{\circ}{ }^{30,36}$ We have therefore studied the effect of $\left[\mathrm{K}^{+}\right]_{o}$ on scroll wave dynamics.

First of all, Fig. 12 shows the effect of $\left[\mathrm{K}^{+}\right]_{o}$ in spiral wave dynamics. We see that an increase of $\left[\mathrm{K}^{+}\right]_{o}$ dramatically increases the wavelength of the spiral wave and decreases the APD. We observe dense spirals [Fig. 12(A)] for low values of the extracellular potassium concentration and sparse spirals for high values [Figs. 12(C) and 12(D)]. For

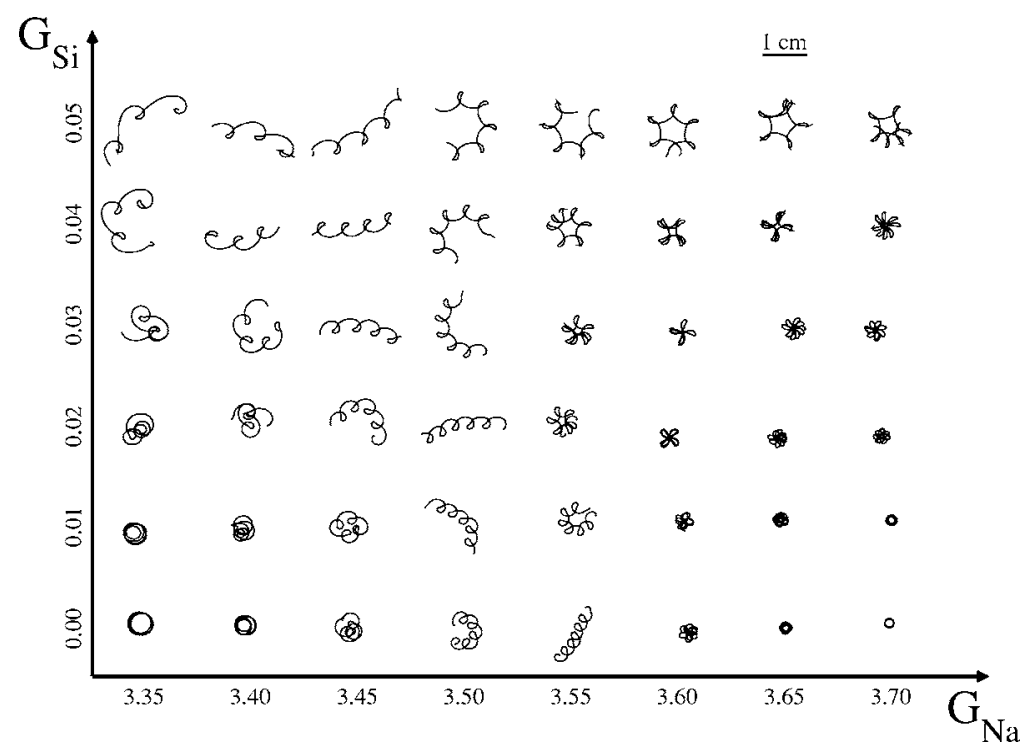

FIG. 11. Phase diagram of the motion of the tip of spiral waves in $2 \mathrm{D}$ cardiac tissue, with $j$ clamped to 1 , in front of the variables $G_{\mathrm{Na}}$ and $G_{\mathrm{Si}}$, with $G_{K}$ $=0.423 \mathrm{mS} / \mathrm{cm}^{2}$. The evolution of the tip is plotted in all the panels for $200 \mathrm{~ms}$. 


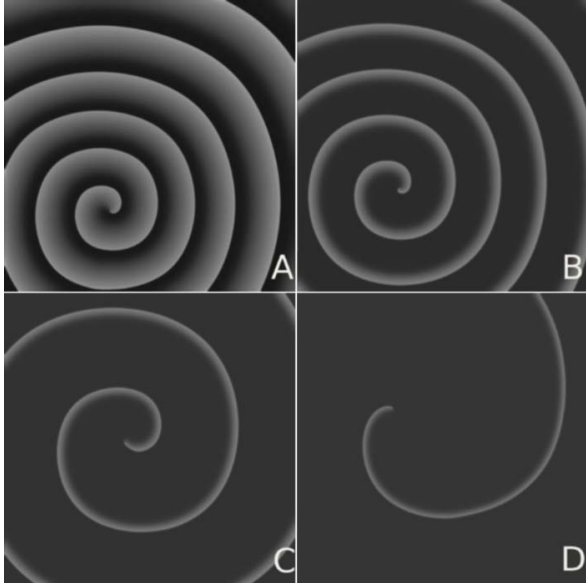

FIG. 12. Examples of spiral waves in cardiac tissue for different values of the extracellular potassium concentration: (A) $\left[\mathrm{K}^{+}\right]_{o}=5.4 \mathrm{mM},(\mathrm{B})\left[\mathrm{K}^{+}\right]_{o}$ $=10.4 \mathrm{mM},(\mathrm{C})\left[\mathrm{K}^{+}\right]_{o}=12.4 \mathrm{mM}$, and (D) $\left[\mathrm{K}^{+}\right]_{o}=13.4 \mathrm{mM}$.

still larger concentrations of the extracellular potassium $\left(\left[\mathrm{K}^{+}\right]_{o}>13.6 \mathrm{mM}\right)$ the cardiac tissue becomes nonexcitable.

Figure 13 shows typical patterns of scroll wave filament drift for the same parameter values as in Fig. 12. We see that the increase in $\left[\mathrm{K}^{+}\right]_{o}$ results in a transition between positive and negative filament tension. For low values of $\left[\mathrm{K}^{+}\right]_{o}$ scroll rings shrink [Figs. 13(A)-13(C)] and, thus, filament tension is positive. Around $\left[\mathrm{K}^{+}\right]_{o} \sim 13.3 \mathrm{mM}$ the tension of the filament changes and scroll rings expand [Fig. 13(D)]. As in the previous cases, the velocity of the scroll ring drift increases when the values of the parameters are close to the boundary between positive and negative filament tension. Indeed, the drift of the scrolls in Figs. 13(C) and 13(D) is larger than in Figs. 13(A) and 13(B); there is also a correspondence between inward meandering and negative filament tension [Figs. 13(D) and 13( $\left.\mathrm{D}^{\prime}\right)$ and between outward meandering and positive tension [Figs. 13(A), 13(B), 13(C), 13( $\left.\mathrm{A}^{\prime}\right)$ $13\left(\mathrm{~B}^{\prime}\right)$, and $\left.13\left(\mathrm{C}^{\prime}\right)\right]$.

One of the consequences of elevating the potassium concentration is the increase of the refractory period, ${ }^{30}$ which
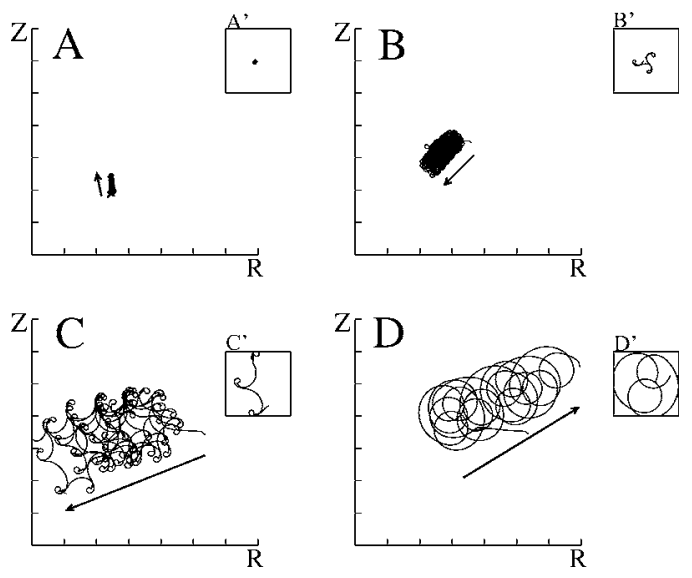

FIG. 13. Dynamics of the filament of scroll rings in the plane $(R, Z)$ for different values of the extracellular potassium concentration: (A) $\left[\mathrm{K}^{+}\right]_{0}$ $=5.4 \mathrm{mM}$, (B) $\left[\mathrm{K}^{+}\right]_{o}=12.4 \mathrm{mM}$, (C) $\left[\mathrm{K}^{+}\right]_{o}=13.0 \mathrm{mM}$, and (D) $\left[\mathrm{K}^{+}\right]_{o}$ $=13.4 \mathrm{mM}$. Small panels show the trajectories of the tip of $2 \mathrm{D}$ spiral waves $(x, y)$. increases the amplitude of meandering of spiral waves. Spiral waves for normal $\left[\mathrm{K}^{+}\right]_{o}$ concentration rotate with a small meandering [Fig. $\left.13\left(\mathrm{~A}^{\prime}\right)\right]$, but as the value of $\left[\mathrm{K}^{+}\right]_{o}$ increases, the meander amplitude grows $\left[\right.$ Fig. $\left.13\left(\mathrm{C}^{\prime}\right)\right]$ although the APD decreases [Fig. 2].

\section{DISCUSSION}

Negative filament tension has been observed in several low-dimensional models of excitable media and reduced models of cardiac tissue. This instability gives rise to chaotic dynamics in 3D media and can potentially be applied to understanding the mechanisms of ventricular fibrillation.

The LR1 model has been widely used to study the dynamics of cardiac cells and the propagation of waves in cardiac tissue. We have demonstrated by numerical simulations that negative filament tension exists in the LR1 model of cardiac tissue. This instability leads to chaotic wave dynamics in a slab of cardiac tissue. The character of the 3D turbulence obtained with the LR1 model does not substantially differ from the chaotic behaviors obtained with simpler models.

Negative filament tension is only observed in a parameter region where excitability is weak and near the limit of propagation failure. The propagation of waves in cardiac tissue is usually observed in the limit of normal or high excitability; i.e., far from this region of parameters. However, there are some conditions in which excitability of cardiac tissue is substantially decreased; for example, during ischemia. We have mimicked the conditions of ischemia by increasing the concentration of extracellular potassium and found, that combined with decreasing of sodium and slow inward currents, it may result in the onset of instability.

Although negative filament tension was found in a relatively small region of parameters, we think that it still can be observed in cardiac tissue in ischemic conditions. Indeed, the tissue excitability during the course of ischemia changes from normal to pathological conditions where the tissue is unable to sustain propagating waves. Therefore, as follows from our simulations, at a certain moment of time just before propagation failure the tissue should be in the region of the negative filament tension regime. In addition, ischemia is not homogeneous in space, thus the conditions for negative filament tension can be satisfied locally in different regions at different times.

Another effect of ischemia is decreasing of cell-to-cell coupling. ${ }^{37}$ Such decreasing can be modeled by decreasing the value of the diffusion coefficient in Eq. (3). In general, change of the diffusion coefficient does not changes the regime of filament drift, because it can be rescaled from the equations by changing the spatial variables. However, if cellto-cell uncoupling will be substantial, it will lead to discrete propagation, ${ }^{37}$ and thus to additional decrease of excitability of the tissue, which will increase the region of existence of the expansion regime.

It would be interesting to look for a correlation of the onset of arrhythmias and tissue excitability in ischemic conditions. The results of this paper suggest that if such arrhythmias will occur close to propagation failure, they are likely 
to have a negative filament tension mechanism.

Another pure 3D instability, is scroll wave breakup due to rotational anisotropy of cardiac tissue. ${ }^{29,38}$ As was shown in Ref. 29, such instability occurs as a result of filament elongation due to the twist of the filament that develops because of tissue anisotropy. Anisotropy should also affect filaments in the negative filament tension regime, thus it should enhance the instability. Therefore, we can expect that taking into account anisotropy of cardiac tissue will increase the region of parameters for which negative filament tension can be observed.

We show that for drift velocities, the transition from the positive to the negative filament tension in LR1 model is not continuous (Fig. 6). Similar behavior for the normal velocity has already been observed in low-dimensional models of excitable media. ${ }^{20,34}$ In those models the normal velocity shows a maximum in the negative tension region and a minimum in the positive tension region. Between both peaks there is a value of the parameters with null radial velocity corresponding to null filament tension. We cannot reproduce these results with the LR1 model because the large meandering observed in the dynamics of the filament masks these drifts. The behavior of the binormal velocity, however, differs from the previous results obtained with reduced models of excitable media. This change between positive and negative drift has never been observed.

In all cases, we observed a clear correlation between inward petals on the meandering of spiral waves and negative filament tension. We propose to use it as a $2 \mathrm{D}$ test for the negative filament tension regime. Using it we can suggest that negative filament tension should be observed in the Beeler-Reuter model, ${ }^{39}$ where inward meandering has been reported in the weak excitability limit. ${ }^{40}$ Furthermore, inward meandering of spiral waves was found in experiments of $2 \mathrm{D}$ preparations of cardiac tissue. ${ }^{41}$ This suggests that we can expect negative filament tension in thicker $3 \mathrm{D}$ preparations under the same experimental conditions as in Ref. 41.

\section{ACKNOWLEDGMENTS}

We acknowledge the hospitality of the Mathematical Biology Group of the University of Dundee where this research was begun. S.A. acknowledges the EU financial support in the framework of the EU Marie Curie Research and Training Network "Unifying principles in nonequilibrium pattern formation". We are thankful to Dr. K. H. W. J. ten Tusscher for providing software for this research and help in preparation of the manuscript.

${ }^{1}$ R. J. Myerburg, K. M. Kessler, and A. Interian Jr., Cardiac Electrophysiology. From Cell to Bedside, edited by D. P. Zipes and J. Jalife (Saunders, Philadelphia, 1990), p. 666.

${ }^{2}$ A. T. Winfree and S. H. Strogatz, Nature (London) 311, 611 (1984).

${ }^{3}$ F. H. Samie, O. Berenfeld, J. Anumonwo, S. F. Mironov, S. Udassi, J.
Beaumont, S. Taffet, A. M. Pertsov, and J. Jalife, Circ. Res. 89, 1216 (2001)

${ }^{4}$ G. K. Moe, W. C. Rheinbolt, and J. A. Abildskov, Am. Heart J. 67, 200 (1964).

${ }^{5}$ A. V. Panfilov and A. M. Pertsov, Philos. Trans. R. Soc. London, Ser. A 359, 1315 (2001)

${ }^{6}$ F. H. Fenton, E. M. Cherry, H. M. Hastings, and S. J. Evans, Chaos 12, 852 (2002).

${ }^{7}$ A. T. Winfree, Science 266, 1003 (1994).

${ }^{8}$ A. T. Winfree, Science 181, 937 (1973).

${ }^{9}$ A. M. Pertsov, R. R. Aliev, and V. I. Krinsky, Nature (London) 345, 419 (1990).

${ }^{10}$ F. Siegert and C. J. Weijer, Proc. Natl. Acad. Sci. U.S.A. 89, 6433 (1992).

${ }^{11}$ A. M. Pertsov and J. Jalife, Cardiac Electrophysiology. From Cell to Bedside, 2nd ed., edited by D. P. Zipes and J. Jalife (Saunders, Philadelphia, 1995), p, 403

${ }^{12}$ I. R. Efimov, V. Y. Sidorov, Y. Cheng, and B. Wollenzier, J. Cardiovasc. Electrophysiol. 10, 1452 (1999).

${ }^{13}$ M. Wellner, O. Berenfeld, J. Jalife, and A. M. Pertsov, Proc. Natl. Acad Sci. U.S.A. 99, 8015 (2002).

${ }^{14}$ A. V. Panfilov and A. N. Rudenko, Physica D 28, 215 (1987).

${ }^{15}$ V. N. Biktashev, A. V. Holden, and H. Zhang, Philos. Trans. R. Soc. London, Ser. A 347, 611 (1994).

${ }^{16}$ A. V. Panfilov, R. R. Aliev, and A. V. Mushinsky, Physica D 36, 181 (1989).

${ }^{17}$ H. Henry and V. Hakim, Phys. Rev. E 65, 046235 (2002).

${ }^{18}$ P. K. Braznhik, V. A. Davydov, V. S. Zykov, and A. S. Mikhailov, Sov. Phys. JETP 66, 984 (1987).

${ }^{19}$ S. Alonso, F. Sagués, and A. S. Mikhailov, Science 299, 1722 (2003).

${ }^{20}$ S. Alonso, R. Kähler, A. S. Mikhailov, and F. Sagués, Phys. Rev. E 70 056201 (2004).

${ }^{21}$ D. Barkley, M. Kness, and L. S. Tuckerman, Phys. Rev. A 42, 2489 (1990).

${ }^{22}$ C. H. Luo and Y. Rudy, Circ. Res. 68, 1501 (1991).

${ }^{23}$ D. Noble and Y. Rudy, Philos. Trans. R. Soc. London, Ser. A 359, 1127 (2001).

${ }^{24}$ Z. Qu, J. Kil, F. Xie, A. Garfinkel, and J. N. Weiss, Biophys. J. 78, 2761 (2000).

${ }^{25}$ A. Garfinkel and Z. Qu, Cardiac Electrophysiology. From Cell to Bedside, 3rd ed. edited by D. P. Zipes and J. Jalife (Saunders, Philadelphia, 1999), p. 315.

${ }^{26}$ K. H. W. J. Ten Tusscher and A. V. Panfilov, Am. J. Physiol. Heart Circ. Physiol. 284, H542 (2003).

${ }^{27}$ Z. Qu, J. N. Weiss, and A. Garfinkel, Am. J. Physiol. 276, H269 (1999).

${ }^{28}$ Z. Qu, F. Xie, A. Garfinkel, and J. N. Weiss, Ann. Biomed. Eng. 28, 755 (2000).

${ }^{29}$ F. H. Fenton and A. Karma, Chaos 8, 20 (1998).

${ }^{30}$ R. M. Shaw and Y. Rudy, Circ. Res. 35, 256 (1997).

${ }^{31}$ L. Ebihara and E. A. Johnson, Biophys. J. 32, 779 (1980).

${ }^{32}$ A. S. Mikhailov, V. A. Davydov, and V. S. Zykov, Physica D 70, 1 (1994).

${ }^{33}$ V. S. Zykov, Simulation of Wave Processes in Excitable Media (Manchester University Press, Manchester, 1987).

${ }^{34}$ H. Henry, Phys. Rev. E 70, 026204 (2004).

${ }^{35}$ S. Alonso, F. Sagués, and A. S. Mikhailov, J. Phys. Chem. A 110, 12063 (2006).

${ }^{36}$ F. Xie, Z. Qu, A. Garfinkel, and J. N. Weiss, Am. J. Physiol. Heart Circ. Physiol. 280, H1667 (2001).

${ }^{37}$ A. G. Kleber and Y. Rudy, Physiol. Rev. 84, 431 (2004).

${ }^{38}$ A. V. Panfilov and J. P. Keener, Physica D 84, 545 (1995).

${ }^{39}$ G. W. Beeler and H. Reuter, J. Physiol. (London) 268, 177 (1977).

${ }^{40}$ I. R. Efimov, V. I. Krinsky, and J. Jalife, Chaos, Solitons Fractals 5, 513 (1995).

${ }^{41}$ D. T. Kim, Y. Kwan, J. J. Lee, T. Ikeda, T. Uchida, K. Kamjoo, Y. Kim, J. J. C. Ong, C. A. Athill, T. Wu, L. Czer, H. S. Karaguezian, and P. Chen, Chaos 8, 137 (1998) 